\title{
Studies of quarkonia production and polarisation at $\mathrm{LHCb}$
}

\section{Maddalena FROSINI*( Università e INFN (IT))}

E-mail: maddalena.frosiniecern.ch

Studies of quarkonia production in the forward region provide important tests of non-relativistic QCD. The LHCb experiment has collected a dataset corresponding to an integrated luminosity of about $3 \mathrm{fb}^{-1}$ in proton-proton collisions at $\sqrt{s}=7$ and $8 \mathrm{TeV}$. We present studies of the production of the $J / \psi$ and $\chi_{c}$ charmonium states as well as those of $\Upsilon$. Absolute and relative production cross sections are presented and compared to the most recent theoretical predictions when available. We also discuss the future prospects for these measurements.

XXI International Workshop on Deep-Inelastic Scattering and Related Subjects 22-26 April, 2013

Marseilles, France

* Speaker. 


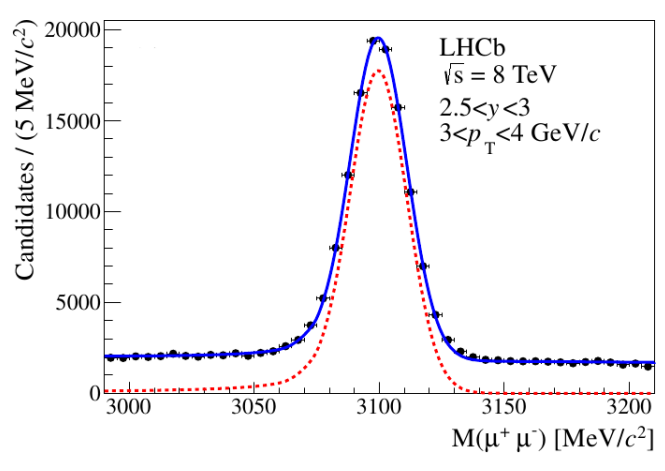

Figure 1: $J / \psi$ mass distribution for a selected $p_{T}$ and $y$ bin.

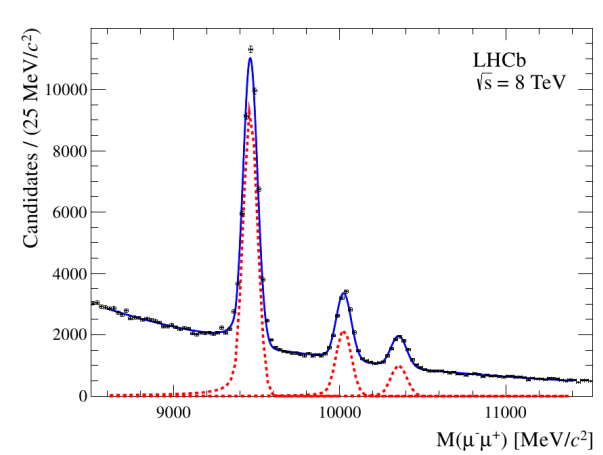

Figure 2: $\Upsilon(1 S), \Upsilon(2 S)$ and $\Upsilon(3 S)$ mass distribution for $p_{T}<15 \mathrm{GeV} / c$ and $2.0<y<4.5$.

\section{Introduction}

Despite the many theoretical models (in particular the Colour Singlet $[1,2]$ and the Colour Octet model [3], based on a NRQCD approach), proposed in the recent years, the quarkonium production mechanism is not yet completely understood. Thanks to the excellent capability, LHCb is contributing with different measurements in understanding the production mechanism.

The LHCb detector [4] is a single-arm forward spectrometer covering the pseudo-rapidity range $2<\eta<5$, designed for the study of hadrons containing $b$ or $c$ quarks. The LHCb detector includes a high precision tracking system, two ring-imaging Cherenkov detectors for the identification of charged hadrons, a calorimeter system and a muon detector.

\section{2. $J / \psi$ and $\Upsilon$ production}

The $J / \psi$ and $\Upsilon$ double differential cross sections have been measured at $\sqrt{s}=8 \mathrm{TeV}$ using two data samples of $18 \mathrm{pb}^{-1}$ and $51 \mathrm{pb}^{-1}$, respectively for the $J / \psi$ and $\Upsilon$ analysis [7]. Using a data sample of $70 \mathrm{nb}^{-1}$ the differential cross section (as a function of transverse momentum) of the inclusive $J / \psi$ is measured at $\sqrt{s}=2.76 \mathrm{TeV}$ [8]. The analysis strategy is the same used for the $J / \psi$ and $\Upsilon$ production measurement at $\sqrt{s}=7 \mathrm{TeV}[5,6]$ and it consists in reconstructing the candidates in their dimuon decays. The selection requires at least one reconstructed primary vertex in each event and the candidates are formed from pairs of opposite sign charged tracks (with a good track fit quality) identified as muons. The number of signal events is extracted from a fit to the invariant mass distribution of the two muons, shown in Figs. 1 and 2, using a Crystal Ball function for the signal peak and an exponential for the background. The prompt and delayed (coming from $b$-hadron decays) $J / \psi$ components are separated using the pseudo proper time, as in [5]. Fig. 3 shows the differential cross section of the prompt $J / \psi$ component, in rapidity range $2.0<y<4.5$, as a function of $p_{T}$. Results are compared with the prediction of different theoretical models, showing in particular a good agreement with the NRQCD predictions [9, 10]. In Fig. 4 the differential cross section of the delayed $J / \psi$ component is shown compared with the FONLL computation $[11,12]$. The total integrated cross sections for $p_{T}<14 \mathrm{GeV} / c$ and $2.0<y<4.5$ are:

$$
\sigma_{\text {prompt }}=10.94 \pm 0.02 \text { (stat) } \pm 0.79 \text { (sys) } \mu \mathrm{b}, \sigma_{d e l}=1.28 \pm 0.01 \text { (stat) } \pm 0.11 \text { (sys) } \mu \mathrm{b} \text {. }
$$




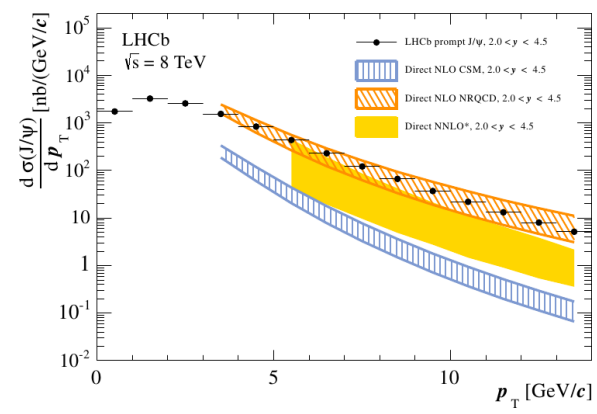

Figure 3: Differential cross section of prompt $J / \psi$ compared with the NRQCD $[9,10]$ and Colour Singlet computation at NNLO* [1] and at NLO [2].

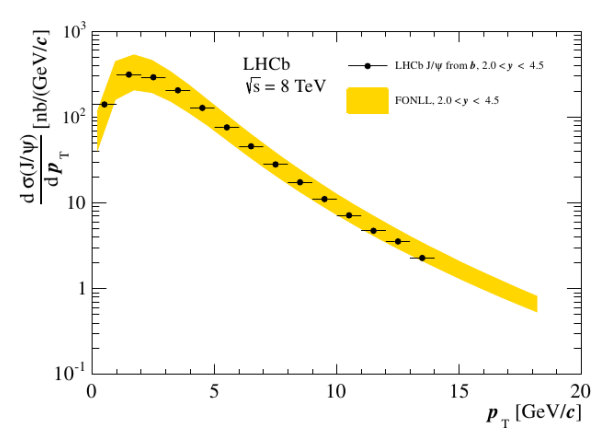

Figure 4: Differential cross section of delayed $J / \psi$ component compared with FONLL computation $[11,12]$.

The differential cross section of inclusive $J / \psi$ measured at $\sqrt{s}=2.76$ is shown in Fig. 5 as a function of $p_{T}$. The integrated inclusive cross section for $p_{T}<12 \mathrm{GeV} / c$ and $2.0<y<4.5$ is:

$$
\sigma_{\text {incl }}=5.6 \pm 0.1(\text { stat }) \pm 0.4(\text { syst }) \mu \mathrm{b} .
$$

In Fig. 6 the total cross section of the delayed $J / \psi$ component is shown as a function of $\sqrt{s}$, using the measurements published by the LHCb collaboration at $2.76 \mathrm{TeV}, 7 \mathrm{TeV}$ and $8 \mathrm{TeV}[8,5,7]$. The experimental values are compared with the FONLL theoretical computation [7], showing a good agreement with the expectation.

In Fig. 7 the differential cross sections of the three $\Upsilon$ states, $\Upsilon(1 S), \Upsilon(2 S)$ and $\Upsilon(3 S)$ are shown as a function of $p_{T}$. Experimental results are compared with the Colour Singlet calculation at NLO [2] and NNLO* [13]. The integrated values for $p_{T}<15 \mathrm{GeV} / c$ and $2.0<y<4.5$ are:

$$
\begin{aligned}
& \sigma_{\Upsilon(1 S)} \cdot \mathscr{B}^{1 S}=3.241 \pm 0.018(\text { stat }) \pm 0.231 \text { (syst) } \mathrm{nb}, \\
& \sigma_{\Upsilon(2 S)} \cdot \mathscr{B}^{2 S}=0.761 \pm 0.008 \text { (stat) } \pm 0.055(\text { syst }) \mathrm{nb}, \\
& \sigma_{\Upsilon(3 S)} \cdot \mathscr{B}^{3 S}=0.369 \pm 0.005 \text { (stat) } \pm 0.027 \text { (syst) nb, }
\end{aligned}
$$

where $\mathscr{B}^{i S}$ are the branching ratios of $\Upsilon(i S)$ state decaying in two muons.

\section{3. $\chi_{c}$ production}

The study of the $J / \psi$ production through the radiative decays of the $\chi_{c}$ states provides a useful test of both the Color Singlet and Color Octet model. The relative cross section $\sigma\left(\chi_{c 2}\right) / \sigma\left(\chi_{c 1}\right)$ is measured using two different data sample of $37 \mathrm{pb}^{-1}$ [14] and $370 \mathrm{pb}^{-1}$ [15], acquired during the 2010 and 2011 at $\sqrt{s}=7 \mathrm{TeV}$. The $\chi_{c}$ states are identified through their radiative decay $\chi_{c} \rightarrow J / \psi \gamma$ with the $J / \psi$ decaying in two muons. For the first measurement, $\chi_{c}$ states are reconstructed using photons detected in the calorimeter system: this allows to have a higher statistics but the poor resolution of the calorimeter makes the separation of the $\chi_{c 1}$ and $\chi_{c 2}$ peaks difficult. The second measurement has been performed using the converted photons in the detector material before the magnet: this allows to resolve the two states, thanks to the good resolution of the tracker.

In both measurements the efficiency is determined from the Monte Carlo simulation and the number 


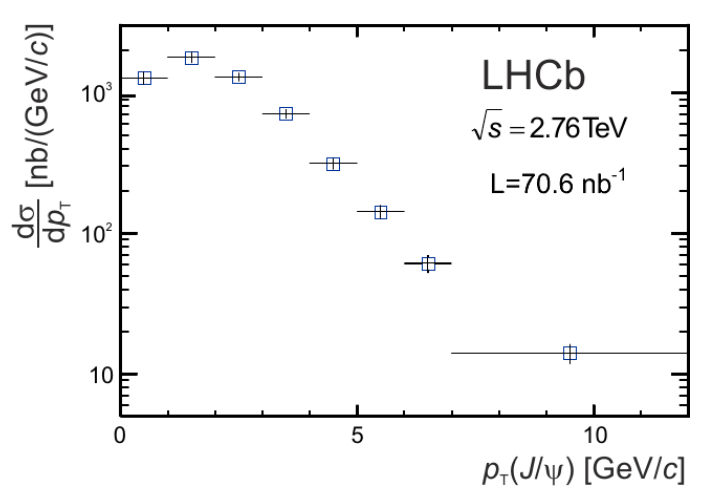

Figure 5: Differential cross section of the inclusive $J / \psi$.

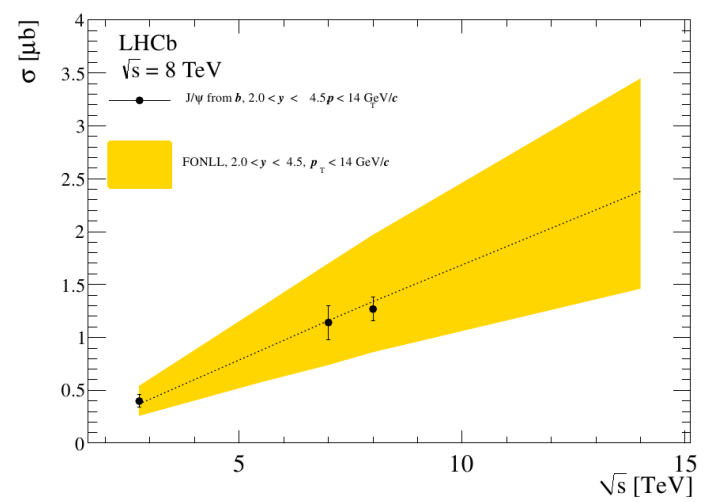

Figure 6: Total cross section of $J / \psi$ from $b$ component as a function of $\sqrt{s}$. Experimental results are compared with the FONLL prediction $[11,12]$.
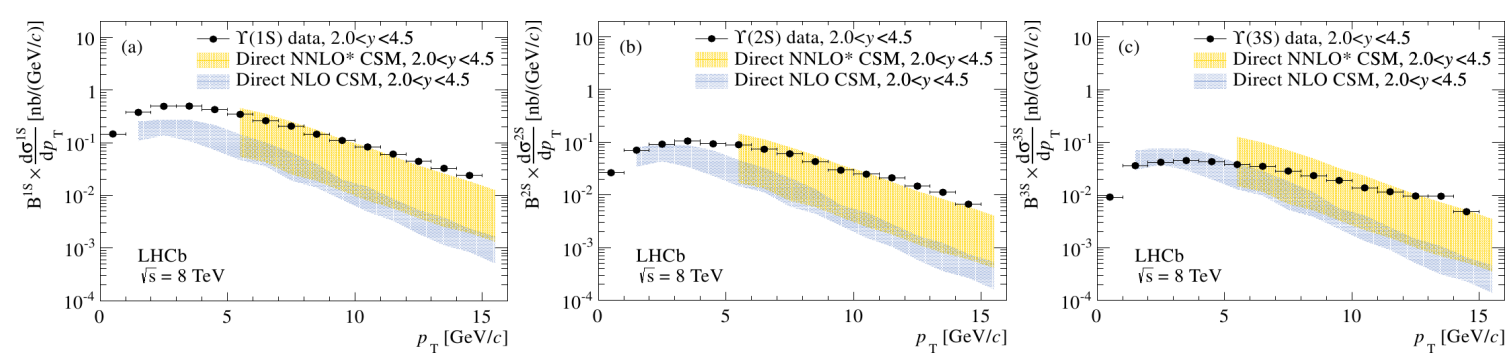

Figure 7: Differential cross section of $\Upsilon(1 S), \Upsilon(2 S)$ and $\Upsilon(3 S)$ compared with the Colour Singlet computation at NLO [2] and NNLO* [13].

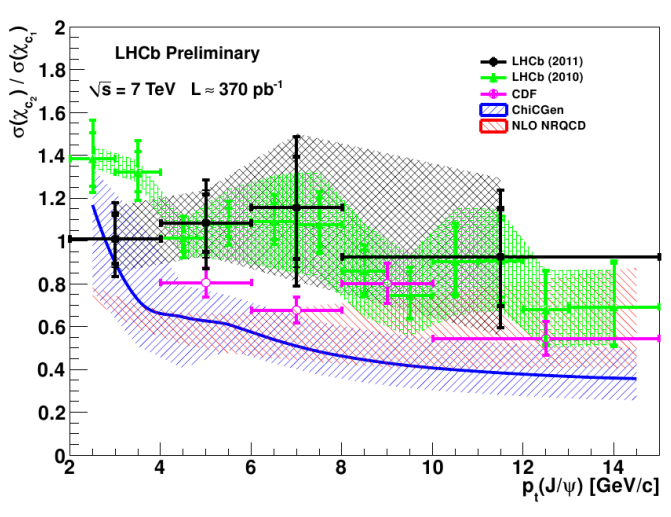

Figure 8: Relative cross section $\sigma\left(\chi_{c 2}\right) / \sigma\left(\chi_{c 1}\right)$ in bins of $J / \psi$ transverse momentum.

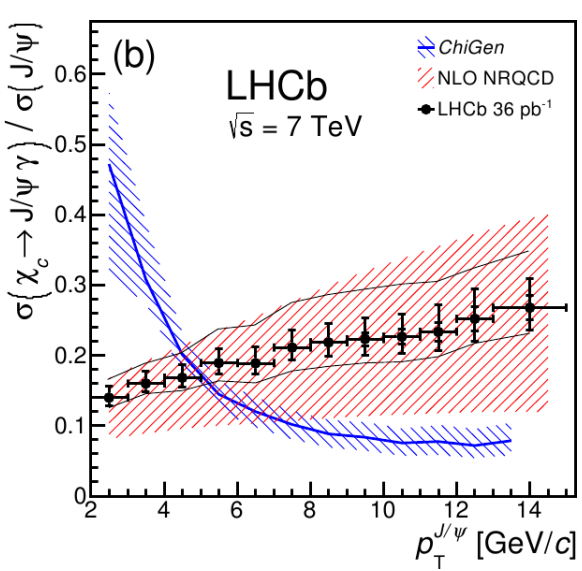

Figure 9: Relative cross section $\sigma\left(\chi_{c}\right) / \sigma(J / \psi)$ in bins of $J / \psi p_{T}$, compared with the Colour Singlet (blue) and Colour Octet (red) predictions. 

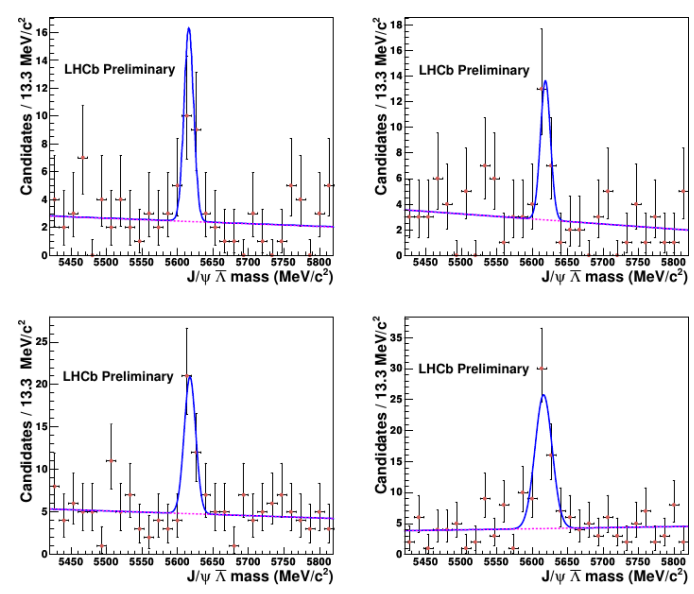

Figure 10: Mass distribution of $\bar{\Lambda}_{b}^{0}$ mass splitted according to the $\bar{\Lambda}_{b}^{0}$ decay flight (top/bottom plots) and magnet polarity (left/right plots).

of signal events is extracted with a fit to the invariant mass difference spectra.

The relative cross section results $\sigma\left(\chi_{c 2}\right) / \sigma\left(\chi_{c 1}\right)$ obtained in 2010 and 2011, are shown in Fig.8 respectively in green and black. Experimental results are compared to the Color Singlet [16] and NRQCD [17] predictions, and to the CDF measurement [18]. The shaded area represents the maximum effect due to the unknown $\chi_{c}$ polarization.

The $\chi_{c}$ to $J / \psi$ ratio has been measured with a $36 \mathrm{pb}^{-1}$ data sample [19]. Also in this case the $\chi_{c}$ states are reconstructed through their radiative decay to the $J / \psi$ with the $J / \psi$ decaying into two muons. The results are shown in Fig.9, compared with the Color Singlet [16] and NRQCD expectation [17], showing a good agreement with the last one.

\section{4. $\Lambda_{b}$ production}

LHCb has presented recently a preliminary result on the $\Lambda_{b}^{0}$ in the decay channel $\Lambda_{b}^{0} \rightarrow J / \psi \Lambda$, with $J / \psi \rightarrow \mu^{+} \mu^{-}$in the region $p_{T}<13 \mathrm{GeV} / c$ and $2.2<y<4.5$ [20]. The measurement is based on a data sample of $36 \mathrm{pb}^{-1}$ of $p p$ collisions at $\sqrt{s}=7 \mathrm{TeV}$ recorded by the experiment in 2010. The data sample has been splitted in eight categories according to the $b$ quark flavour, the magnet polarity and the decay flight of the $\Lambda_{0}$, and the mass distribution are shown in Fig. 10. The averaged results for the $\Lambda_{b}^{0}$ and $\bar{\Lambda}_{b}^{0}$ are respectively

$$
\begin{aligned}
& \sigma_{\Lambda_{b}^{0}} \times \mathscr{B}\left(\Lambda_{b} \rightarrow \Lambda^{0} J / \psi\right)=4.08 \pm 0.59 \text { (stat) } \pm 0.36 \text { (sys) } \mu \mathrm{b} \\
& \sigma_{\bar{\Lambda}_{b}^{0}} \times \mathscr{B}\left(\bar{\Lambda}_{b} \rightarrow \bar{\Lambda}^{0} J / \psi\right)=2.60 \pm 0.46 \text { (stat) } \pm 0.26 \text { (sys) } \mu \mathrm{b} .
\end{aligned}
$$

The results are qualitatively in agreement with the CMS measurement [21], even if it is not possible to perform a quantitative comparison because of the different kinematic range of the two analyses.

\section{Conclusion}

In the present paper a few selected results obtained by $\mathrm{LHCb}$ in the quarkonia sector have been presented. More other interesting measurements have been published by the collaboration, 
mentioning for example the $\psi(2 S)$ production [22], the double $J / \psi[23]$ and $J / \psi$ production in association with an open charm [24], and the measurement of the fraction of $\Upsilon$ coming from $\chi_{b}$ decays [25]. The measurement of $J / \psi$ polarization is also ongoing and is expected to be published soon.

\section{References}

[1] J. Lansberg, On the mechanisms of heavy-quarkonium hadroproduction, Eur. Phys. J. C 61 (2009) 693, [hep-ph/0811.4005]

[2] J.M. Campbell, F. Maltoni and F. Tramontano, $Q C D$ corrections to $J / \psi$ and $\Upsilon$ production at hadron colliders, Phys. Rev. Lett. 98 (2007) 252002, [hep-ph/0703113]

[3] G. T. Bodwin., E. Braaten, G.P. Lepage, Rigorous QCD analysis of inclusive annihilation and production of heavy quarkonium, Phys. Rev. D 51 (1995) 1125

[4] LHCb Collaboration, The LHCb Detector at the LHC JINST 3 S08005 (2008)

[5] LHCb Collaboration, Measurement of $J / \psi$ production in pp collisions at $\sqrt{s}=7 \mathrm{TeV}$, Eur. Phys. J. C 71 (2011) 1645, [hep-ex/1103.0423]

[6] LHCb Collaboration, Measurement of $\Upsilon$ production in pp collisions at $\sqrt{s}=7 \mathrm{TeV}$, Eur. Phys. J. C 72 (2012) 2025, [hep-ex/1202.6579]

[7] LHCb Collaboration, Production of $J / \psi$ and $\Upsilon$ mesons in pp collisions at $\sqrt{s}=8 \mathrm{TeV}$, JHEP 06 (2013) 064, [hep-ex/1304.6977]

[8] LHCb Collaboration, Measurement of $J / \psi$ production in pp collisions at $\sqrt{s}=2.76 \mathrm{TeV}$, JHEP 1302 (2013) 041, [hep-ex/1212.1045]

[9] M. Butenschöen and B.A. Kniehl, World data of $J / \psi$ production consolidate NRQCD factorization at NLO, Phys. Rev. D 84 (2011) 051501, [hep-ph/1105.0820]

[10] M. Butenschöen and B.A. Kniehl, Reconciling J/ $\psi$ production at HERA, RHIC, Tevatron and LHC with NRQCD factorization at next-to-leading order, Phys. Rev. Lett. 106 (2011) 022003 , [hep-ph/1009.5662]

[11] M. Cacciari et al., Theoretical predictions for charm and bottom production at the LHC, JHEP 10 (2012) 137, [hep-ph/1205.6344]

[12] M. Cacciari, M. Greco and P. Nason, The $p_{T}$ spectrum in heavy flavour hadroproduction, JHEP 05 (1998) 007,[hep-ph/9803400]

[13] P. Artoisenet, J.M. Campbell, J. Lansberg, F. Maltoni and F. Tramontano, $\Upsilon$ production at Fermilab Tevatron and LHC energies, Phys. Rev. Lett. 101 (2008) 152001, [hep-ph/0806.3282]

[14] LHCb Collaboration, Measurement of the cross-section ratio $\sigma\left(\chi_{c 2}\right) / \sigma\left(\chi_{c 1}\right)$ for prompt $\chi_{c}$ production at $\sqrt{s}=7 \mathrm{TeV}$ Phys. Lett. B 714 (2012) 215, [hep-ex/1202.1080]

[15] LHCb Collaboration, Measurement of the relative cross-section $\sigma\left(\chi_{c 2}\right) / \sigma\left(\chi_{c 1}\right)$ of prompt $\chi_{c}$ mesons at $\mathrm{LHCb}$, LHCb-CONF-2011-062

[16] L.A. Harland Lang, W.J. Stirling, http://projects.hepforge.org/superchic/chigen.html

[17] Y.-Q. Ma, K. Wang, K.-T. Chao, QCD radiative corrections to cJ production at hadron colliders Phys. Rev. D 83 (2011) 111503 R, [hep-ph/1002.3987] 
[18] CDF Collaboration, Measurement of $\sigma_{\chi_{c 2}} B\left(\chi_{c 2} \rightarrow J / \psi \gamma\right) / \sigma_{\chi_{c 1}} B\left(\chi_{c 1} \rightarrow J / \psi \gamma\right)$ in $p \bar{p}$ collisions at $\sqrt{s}=1.96$ TeV, Phys. Rev. Lett. 98 (2007) 232001, [hep-ex/0703028]

[19] LHCb Collaboration, Measurement of the ratio of prompt $\chi_{c}$ to $J / \psi$ production in pp collisions at $\sqrt{s}=7 \mathrm{TeV}$ Phys. Lett. B 718 (2012) 431, [hep-ex/1204.1462]

[20] LHCb Collaboration, Studies of $\Lambda_{b}^{0}$ production in pp collisions at $\sqrt{s}=7 \mathrm{TeV}$, LHCb-CONF-2012-031

[21] CMS Collaboration, Measurement of the $\Lambda_{b}$ cross section and the $\bar{\Lambda}_{b}$ to $\Lambda_{b}$ ratio with $\Lambda_{b}$ to $J / \psi \Lambda$ decays in pp collisions at $\sqrt{s}=7$ TeV, Phys. Lett. B 714 (2012), 136, [hep-ex/1205.0594]

[22] LHCb Collaboration, Measurement of $\psi(2 S)$ meson production in pp collisions at $\sqrt{s}=7 \mathrm{TeV}$, Eur. Phys. J. C 72 (2012) 2100, [hep-ex/1204.1258]

[23] LHCb Collaboration, Observation of $J / \psi$ pair production in pp collisions at $\sqrt{s}=7$ TeV, Phys. Lett. B 707 (2012) 52, [hep-ex/1109.0963 ]

[24] LHCb Collaboration, Observation of double charm production involving open charm in pp collisions at $\sqrt{s}=7 \mathrm{TeV}$, JHEP 06 (2012) 141, [hep-ex/1205.0975]

[25] LHCb Collaboration, Measurement of the fraction of $\Upsilon(1 S)$ originating from $\chi_{b}(1 P)$ decays in $p p$ collisions at $\sqrt{s}=7 \mathrm{TeV}$, JHEP 11 (2012) 031, [hep-ex/1209.0282] 